\title{
Neurophotonics
}

\section{Evaluation of rigid registration methods for whole head imaging in diffuse optical tomography}

Xue Wu

Adam T. Eggebrecht

Silvina L. Ferradal

Joseph P. Culver

Hamid Dehghani

\section{SPIE.}




\title{
Evaluation of rigid registration methods for whole head imaging in diffuse optical tomography
}

\author{
Xue Wu, ${ }^{a}$ Adam T. Eggebrecht, ${ }^{\mathrm{b}}$ Silvina L. Ferradal, ${ }^{\mathrm{c}}$ Joseph P. Culver, ${ }^{\mathrm{b}, \mathrm{d}}$ and Hamid Dehghani ${ }^{\mathrm{a}, \star}$ \\ aUniversity of Birmingham, School of Computer Science, Edgbaston, Birmingham B15 2TT, United Kingdom \\ ${ }^{b}$ Washington University School of Medicine, Department of Radiology, 4525 Scott Avenue, St. Louis, Missouri 63110, United States \\ ${ }^{\mathrm{c}}$ Fetal-Neonatal Neuroimaging and Developmental Science Center, Boston Children's Hospital, 300 Longwood Avenue, Boston, \\ Massachusetts 02115, United States \\ 'Washington University, Department of Biomedical Engineering, One Brookings Drive, St. Louis, Missouri 63130, United States
}

\begin{abstract}
Functional brain imaging has become an important neuroimaging technique for the study of brain organization and development. Compared to other imaging techniques, diffuse optical tomography (DOT) is a portable and low-cost technique that can be applied to infants and hospitalized patients using an atlasbased light model. For DOT imaging, the accuracy of the forward model has a direct effect on the resulting recovered brain function within a field of view and so the accuracy of the spatially normalized atlas-based forward models must be evaluated. Herein, the accuracy of atlas-based DOT is evaluated on models that are spatially normalized via a number of different rigid registration methods on 24 subjects. A multileveled approach is developed to evaluate the correlation of the geometrical and sensitivity accuracies across the full field of view as well as within specific functional subregions. Results demonstrate that different registration methods are optimal for recovery of different sets of functional brain regions. However, the "nearest point to point" registration method, based on the EEG 19 landmark system, is shown to be the most appropriate registration method for image quality throughout the field of view of the high-density cap that covers the whole of the optically accessible cortex. (c) The Authors. Published by SPIE under a Creative Commons Attribution 3.0 Unported License. Distribution or reproduction of this work in whole or in part requires full attribution of the original publication, including its DOI. [DOI: 10.1117/1.NPh.2.3.035002]
\end{abstract}

Keywords: diffuse optical tomography; functional connectivity brain imaging; whole head imaging; atlas-based tomography; sensitivity analyses; registration.

Paper 15003PRR received Jan. 20, 2015; accepted for publication Jun. 18, 2015; published online Jul. 21, 2015.

\section{Introduction}

Functional brain imaging techniques such as positron emission tomography (PET) or functional magnetic resonance imaging (fMRI) can measure the physiological activities within the human brain to localize functional activation in response to, for example, visual or auditory stimuli. These techniques measure changes in neurophysiological parameters such as the cerebral blood flow (CBF) or blood-oxygen-level-dependent (BOLD) signal during the brain activation ${ }^{1-4}$ or while it is at rest. ${ }^{5,6}$ The cortex can be divided into different functional regions, such as visual and motor areas, and the functional connectivity between regions can be computed as the correlation between the time courses of the various brain regions. ${ }^{3,7,8}$ This has become an important tool for the study of brain organization and development in health and disease and is applicable to subjects who are unable to respond to tasks such as infants or unconscious patients.

Previous studies have shown that brain activation tasks such as inhibiting reflexive saccades task and hierarchical language tasks are correlated across multiple brain regions. ${ }^{9,10}$ Some neurodevelopmental disorders such as Alzheimer's disease, schizophrenia and adolescent depression have also been shown to be related to the distributed brain networks. ${ }^{11-14}$ Functional connectivity brain imaging is focused on the correlation between

*Address all correspondence to: Hamid Dehghani, E-mail: h.dehghani@cs bham.ac.uk diverse brain regions and mapping of the functional networks. Traditional task-based functional imaging may not be suitable for some subjects such as unconscious patients and infants. Resting-state functional connectivity imaging provides a taskless approach to analyze the correlation between diverse brain regions during spontaneous activity and mapping the restingstate networks. ${ }^{10,15}$ Wide field imaging assesses brain activation from multiple functional regions simultaneously and can be used for both task-based functional connectivity and restingstate functional connectivity imaging.

PET and fMRI are two of the most commonly used imaging techniques for quantitative recovery of brain activity. The brain activities can be monitored using PET, which is based on the changes in the regional $\mathrm{CBF}^{1,8,16,17}$, and using fMRI, which is based on the changes in the BOLD signal. ${ }^{2,3,18}$ However, PET is contraindicated in pediatric patients because of the exposure to ionizing radiation, and fMRI is not permitted with pacemakers and cochlear implants because of the exposure to the strong magnetic fields and the induced electric fields. Additionally, the conventional imaging units of PET and fMRI may cause discomfort for some patients with claustrophobia and may not be suitable for extremely obese patients.

Functional near-infrared spectroscopy (fNIRS) is a nearinfrared light (NIR)-based technique which can be used to monitor and map activations in the human brain by measuring the tissue hemodynamics and oxidative metabolism in the cortex area. ${ }^{19}$ The accuracy of fNIRS recovery, including the effect of the registration methods in fNIRS, has been investigated in 
previous studies. ${ }^{20-23}$ However, fNIRS generally lacks spatial information, which is a clear limitation in the analysis of brain activation and human cortex. Diffuse optical tomography (DOT) is a three-dimensional NIR-based imaging technique that has shown its ability to recover brain function for an adult within a 20-mm depth of the cortex surface by monitoring changes in oxygenated hemoglobin and deoxygenated hemoglobin based on the measures of transmitted/reflected NIR signal from the scalp. ${ }^{24}$ DOT is a nonionizing imaging technique with a portable and low-cost application that can be applied to infants and hospitalized patients and has the potential to monitor the brain activities in real time. ${ }^{10}$

The DOT brain image recovery technique from measured NIR data can be divided into two steps: (1) the generation of a model which simulates the light propagation within the human head, and (2) an inverse process for the recovery of the brain activities which itself is based on the forward model and the measured NIR data. Previous studies of DOT recovery have largely relied on the use of a homogeneous head model derived from the geometry of the head surface; however, the recovered results have generally demonstrated a low accuracy because of the lack of internal structural information. ${ }^{25}$ DOT recovery based on a subject-specific model is a more accurate approach, but it requires structural images from other techniques such as MRI that are not always available. ${ }^{10,26,27}$ On the other hand, atlas-based DOT recovery has proven to be an acceptable alternative when a subject-specific model is not available. ${ }^{28-30}$ An atlas-based head model, generated via a surface-based rigid registration between an atlas and the subject head surfaces, is used as the forward model for atlas-based DOT, the accuracy of which can directly affect the recovery of brain activation.

In DOT brain activation recovery, the measured NIR data on the surface of the head is related linearly to the small changes in internal optical properties via a sensitivity matrix (also known as a Jacobian matrix or weight matrix), which contains a set of values defined as the sensitivity of the measured NIR data to a small change in optical property (details in Sec. 2.3). The analysis of this sensitivity map within the head model can be used to evaluate the accuracy of the forward model for atlas-based DOT. Previous studies of whole head sensitivity analysis in DOT have included the effect of the source-and-detector location on the sensitivity of NIR data to different brain regions. The study of Cooper et al. ${ }^{29}$ on whole head sensitivity analysis uses a source-and-detector array, which covers the visual, auditory and motor functional brain regions to distinguish the highly sensitive areas of the subject's brain accessible to the source-anddetector array. The study of Giacometti et al. ${ }^{31}$ on whole head sensitivity analysis uses a whole head source-and-detector cap based on an EEG 10/5 landmark system and evaluates the overall sensitivity of the whole cortex and the sensitivity in different brain regions based on a contrast-to-noise ratio analysis. This study showed that most brain regions have a relatively high sensitivity $(>50 \%)$ for DOT, though some regions presented lower sensitivity due to the variation in skull and scalp thickness.

In this paper, a whole head sensitivity analysis of DOT is used for the evaluation of atlas-based DOT. The atlas-based head models are generated using a number of different rigid registration methods. The overall sensitivity of the whole adult cortex within the field of view (typically 20-mm deep given the high-density (HD) source-detector configuration used in this study) and the sensitivity value in different brain regions using the atlas-based model and subject-specific model are evaluated and compared. The correlations of the geometrical and sensitivity accuracies for different regions are evaluated.

\section{Methods}

The simulation of NIR light propagation in the human head can be achieved using an anatomical model of the subject. In this study, a finite element model (FEM) of the head having multiple regions is used as the forward model using the NIRFAST software package which uses the diffusion approximation with index-mismatched type III boundary condition. ${ }^{32}$ A subjectspecific model requires anatomical information of the subject head, often obtained from structural MRI. When the MRI is not available, the geometry and internal structures from an atlas-based model can be used as an alternative, ${ }^{28-30}$ generated by registering an atlas model to the subject.

\subsection{Layered Head Mesh}

For both subject-specific and the atlas-based models, the forward model is generated using a segmenting-meshing process of the MRI of the subject or the atlas. The segmenting-meshing process can be divided into three steps. First, MRI scans from the atlas model or a given subject is segmented into five tissue types (skin, bone, CSF, gray, and white matter) by the statistical parametric mapping (SPM) software package based on the tissue distribution probability maps and the pixel intensity of the MRI scans. ${ }^{33,34}$ Second, five masks are generated based on the five-region-segmented scans using the NIRVIEW ${ }^{35}$ and NIRFAST software packages. ${ }^{32}$ Third, layered FEM volumetric meshes are created based on the five masks by NIRFAST and the optical properties are assigned to each node in the mesh, based on its tissue type. Although the optical properties for each tissue type may vary for individual subjects, the same set of heterogeneous optical properties is applied to all of the individual meshes in this study to ensure the consistency of the evaluation and comparison. The set of optical properties used in this study at $750 \mathrm{~nm}$ are based on the previous works which are commonly used as shown in Table 1. ${ }^{36-39}$

\subsection{Atlas-Based Models}

The generation process of atlas-based models in this work relies on a surface-based rigid registration of the atlas mesh to the subject and is summarized in Fig. 1. Based on the segmenting-meshing process outlined above, an atlas mesh and a subject-specific mesh from each subject MRI scan are generated separately. The surfaces of the two meshes are then extracted

Table 1 Head tissue optical properties at $750 \mathrm{~nm}$.

\begin{tabular}{lc}
\hline & $\mu_{a}\left(\mathrm{~mm}^{-1}\right) / \mu_{s}^{\prime}\left(\mathrm{mm}^{-1}\right) /$ refractive index \\
\hline Scalp & $0.0170 / 0.74 / 1.33$ \\
Skull & $0.0116 / 0.94 / 1.33$ \\
CSF & $0.004 / 0.3 / 1.33$ \\
Gray matter & $0.0180 / 0.84 / 1.33$ \\
White matter & $0.0167 / 1.19 / 1.33$ \\
\hline
\end{tabular}




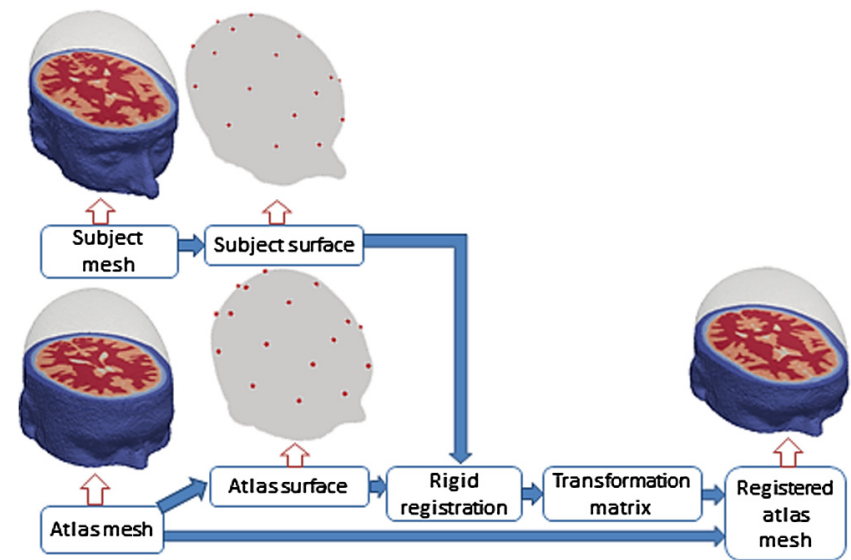

Fig. 1 Workflow of creating a registered atlas-based mesh.

and registered together. The registered atlas mesh is then transformed by applying the affine transformation matrix generated in the registration process to the original atlas mesh. The registered atlas mesh is then used as the atlas-based head mesh in this study.

Registration methods used in this study are focused on the head surface-based rigid registration. Although nonrigid registration methods have also been used for the registration of atlas-based DOT brain imaging, most require some internal structural information of the subject, which is often not available. Nonrigid registration methods can also be applied using external landmarks; however, since nonrigid registration is more localized than rigid registration, it tends to require more fiducial markers, and it can be more computationally intensive..$^{30,40}$ Therefore, rigid registration methods based only on external landmarks are used in this study. The registration process can be divided into two steps. First, external fiducial point sets (landmarks) are extracted from the surfaces of the atlas and the subject mesh, based on the same landmark system. Second, the minimization of the distance between the two landmarks sets is processed based on an optimization algorithm. The affine transformation matrix, which is used to transform from the atlas space to the subject space, is generated for the registration process.

Different registration methods can be used based on different landmark systems or different optimization algorithms. The registration methods used specifically in this study are created based on four different landmark systems and three different optimization algorithms as well as one line-fitting-based registration (Fig. 2). The basic-4-landmark system contains fiducial points extracted manually from four anatomically specified points: the nasion, the inion, and the two temples. EEG 19 and EEG 40 landmark systems contain 19 and 40 landmarks extracted based on the EEG 10/20 system and EEG 10/5 system. A full-head-landmark system contains 700 landmarks extracted uniformly across the whole head surface area under the sourceand-detector cap (details in Sec. 2.4). The basic-4-landmarkbased registration method generates the transformation matrix based on a noniterative optimization algorithm using the corresponding relationship between the two sets of landmarks from the subject and the registration target [the noniterative point to point (nP2P) optimization algorithm]. The $\mathrm{nP} 2 \mathrm{P}$ algorithm is also used in the registration algorithm based on EEG 19, EEG 40, and full-head landmark systems. An iterative optimization algorithm using the corresponding relationship between landmarks sets [point to point (P2P) optimization algorithm] and an iterative optimization algorithm based on the closest point [iterative closest point (ICP) optimization algorithm] is additionally used for EEG 19, EEG 40, and full-head landmark systems. The line-fitting-based registration method generates the transformation matrix by optimizing the fitting of three curves (a temple to temple curve, a nasion to inion curve,

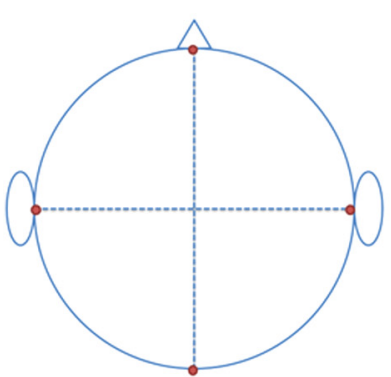

(a)

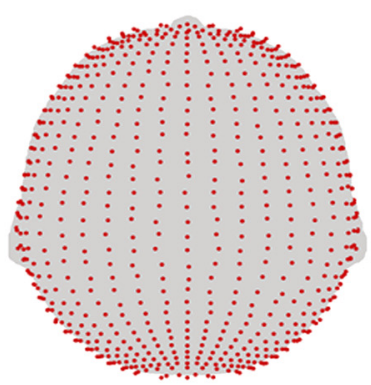

(d)

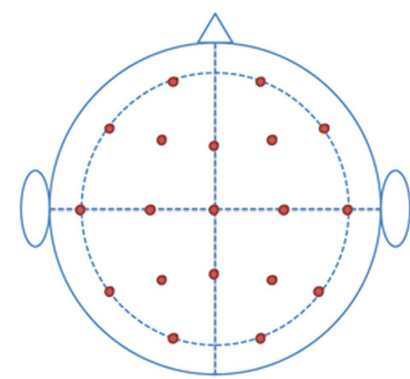

(b)

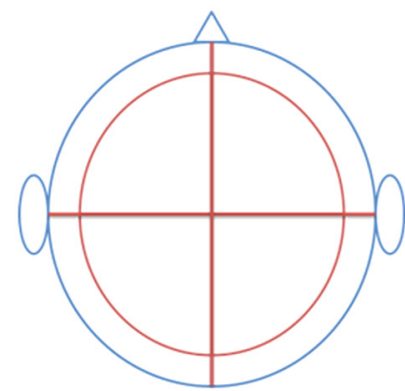

(e)

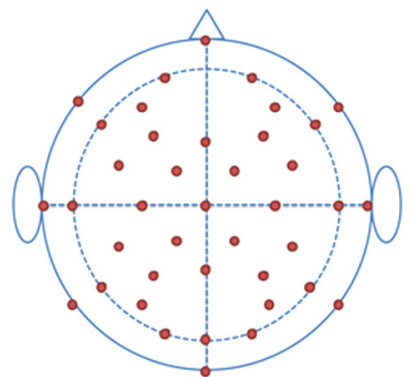

(c)

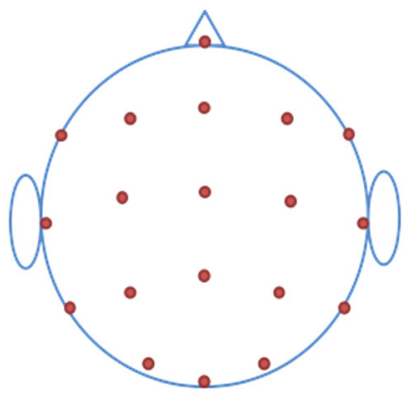

(f)

Fig. 2 Set of different Landmark systems used for registration: (a) basic 4, (b) EEG 19, (c) EEG 40, (d) full head, (e) line, and (f) sphere 19. 
and a circumferential line connecting all four points) as extracted from the head surfaces of the subject and the target. This gives rise to 11 registration methods consisting of basic-4landmark, EEG 19 nP2P, EEG 19 P2P, EEG ICP, EEG 40 nP2P, EEG 40 P2P, EEG 40 ICP, full-head-landmark nP2P, full-headlandmark P2P, full-head-landmark ICP, and line-fitting registration methods, further details of which are covered in depth elsewhere. $^{41}$

Additionally, a spherical coordinate landmark system has also been used which defines a spherical coordinate system based on three fiducial points (the nasion and the left and right temple points) and extracts arbitrary points from the subject scalp as landmarks based on the spherical coordinates. This approach may be considered practically easier to apply than those outlined above. ${ }^{42-44}$ For this purpose, 19 spherical coordinate landmarks using nP2P, P2P, and ICP [Fig. 2(f)] are also used for the registration of the atlas model (named SpnP2p, $\mathrm{SpP} 2 \mathrm{p}$, and SpICP, respectively) and are evaluated and compared with the 11 registration methods outlined above.

\subsection{Sensitivity Matrix for Image Recovery}

The model-based light propagation for brain DOT relies on a forward model which contains the internal structure and optical properties of the subject. The accuracy of the light propagation can be evaluated based on the spatially varying sensitivity of NIR boundary data to the spatially varying optical property. The sensitivity matrix contains the sensitivity of the NIR boundary data of each measurement to the optical property of each mesh node. The sensitivity of NIR boundary data to the optical property can be represented as

$$
J \Delta \mu=\Delta \Phi,
$$

where $\Delta \mu$ is the change in tissue property $\Delta \Phi$, is the change in boundary data, and $J$ is the sensitivity matrix. For continuous wave DOT, the sensitivity matrix is defined as

$$
J=\left[\begin{array}{cccc}
\frac{\partial \ln I_{1}}{\partial \mu_{a 1}} & \frac{\partial \ln I_{1}}{\partial \mu_{a 2}} & \ldots & \frac{\partial \ln I_{1}}{\partial \mu_{a N N}} \\
\frac{\partial \ln I_{2}}{\partial \mu_{a 1}} & \frac{\partial \ln I_{2}}{\partial \mu_{a 2}} & \ldots & \frac{\partial \ln I_{2}}{\partial \mu_{a N N}} \\
\cdots & \cdots & \ldots & \cdots \\
\frac{\partial \ln I_{N M}}{\partial \mu_{a 1}} & \frac{\partial \ln I_{N M}}{\partial \mu_{a 2}} & \cdots & \frac{\partial \ln I_{N M}}{\partial \mu_{a N N}}
\end{array}\right],
$$

where $\ln I$ is the $\log$ amplitude of boundary data, $\mu_{a}$ is the absorption property, $N N$ is the number of nodes, and $N M$ is the number of measurements. The total sensitivity of all measurements at each spatial point of the model is used for the evaluation and comparison of sensitivity accuracy in this study, which is defined as

$J_{\text {total }, n}=\sum_{i=1}^{N M} J_{n, i}$,

where $J_{\text {total }, n}$ is the total sensitivity value at node $n$ for all measurements and $J_{n, i}$ is the sensitivity value of measurement $i$ and node $n$.

\subsection{Simulation Experiments}

For the evaluation of the rigid registration methods for the atlasbased whole head DOT, a simulation experiment is designed based on 14 female and 10 male individual subjects with a mean age of $26( \pm 4)$ and using the ICBM 2009a Nonlinear Symmetric T1w modality atlas model. ${ }^{45,46}$ Subject specific anatomical T1-weighted MPRAGE [echo time (TE) $=3.13 \mathrm{~ms}$, repetition time $(\mathrm{TR})=2400 \mathrm{~ms}$, flip angle $=8 \mathrm{deg}, 1 \times 1 \times$ $1 \mathrm{~mm}^{3}$ isotropic voxels] scans were acquired for each subject (subsequently referred to as T1). All subjects passed MR screening to ensure their safe participation. Informed consent was obtained and the research was approved by the Human Research Protection Office at Washington University School of Medicine. The 5-layer-head meshes with $\sim 400,000$ nodes corresponding to $\sim 2,390,000$ linear tetrahedral elements are generated based on the T1 MRI data of the 24 subjects to provide the subject-specific meshes. The atlas model is then utilized
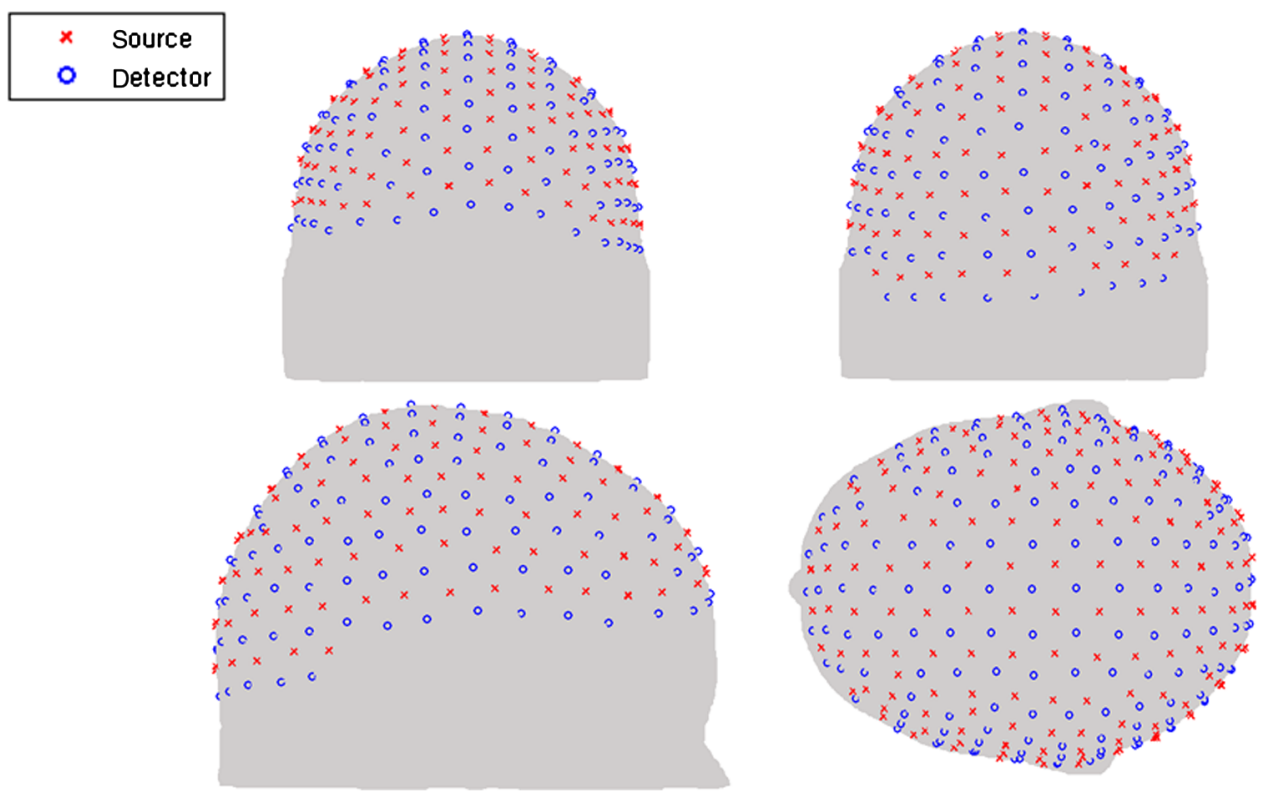

Fig. 3 High-density source-and-detector cap for an example head surface. 
to generate the atlas-based mesh to be used for registration. The atlas mesh is registered to each subject individually using the rigid registration methods outlined above. The optical properties of the five tissue regions in Table 1 are then applied to all of the 336 registered atlas meshes $(24$ subjects $\times 14$ registration methods). An HD source-detector cap with 158 sources and 166 detectors (Fig. 3) is then placed on the surface of all meshes where the sources and detectors in the cap are uniformly distributed on the surfaces of the head and cover the entire surface area above the brain. For all 360 meshes (336 atlas based and 24 subject specific), the sensitivity matrices are then calculated using the first to fourth nearest neighbor measurements at $1.0,2.2,3.0$, and $3.6 \mathrm{~cm}$ source-detector distance on the HD source-detector cap, respectively. ${ }^{47}$

The accuracy of the registration methods can be evaluated by the geometrical accuracy of the registered atlas mesh as compared to the subject-specific mesh. The geometrical accuracy is calculated by the distance from each surface node of the subject-specific mesh to its closest surface node of the registered atlas mesh. The surface region under the HD cap is considered as the region of interest (ROI) for the analyses. The average distances across the head surface within the ROI are calculated based on the registered atlas mesh for all 14 registration methods for all 24 subjects.

The accuracy of the light propagation is evaluated by the accuracy of the sensitivity matrix for each registered atlas mesh. The sensitivity accuracy is calculated by the comparison of sensitivity matrices between the registered atlas mesh and the subject-specific mesh. Specifically, for the evaluation, the sensitivity matrices are generated based on the registered atlas and the subject-specific mesh separately for the HD source-detector cap and then the values within the ROI are selected by utilizing only the sensitivity values on the surface of the cortex which are higher than $1 \%$ of the maximum value. ${ }^{48}$ The total sensitivity is then calculated for all source/detector measurements [Eq. (3)] and these are mapped to the same uniform grid using a linear interpolation function. The total sensitivity values from these two matrices are then compared on this voxel basis.

The correlation of the geometrical and sensitivity error is also evaluated in this study for the analysis of the registration method on the accuracy of light propagation model. Different regions of the head can have different geometry-sensitivity correlations; therefore, a unified analysis based on the EEG 10/20 system region segmentation is used for the evaluation. ${ }^{31}$ This region segmentation is divided into three steps: First, 19 landmarks are extracted from the surface of each head mesh based on the EEG 10/20 system and they are numbered as 1 to 19 . Second, the distance from each node within the mesh to all of the 19 landmarks is calculated and the closest landmark of each node is selected. Third, all the nodes are then labelled based on their closest landmarks and nodes with the same label are considered as the same region. Nineteen regions are then generated based on the EEG 10/20 system. The geometrical and sensitivity error are calculated separately for each region and the correlation is compared for each region.

\section{Results}

\subsection{Evaluation of the Geometry Accuracy}

Each of the considered registration methods is evaluated by the use of a geometrical accuracy analysis of the registered atlas (a)
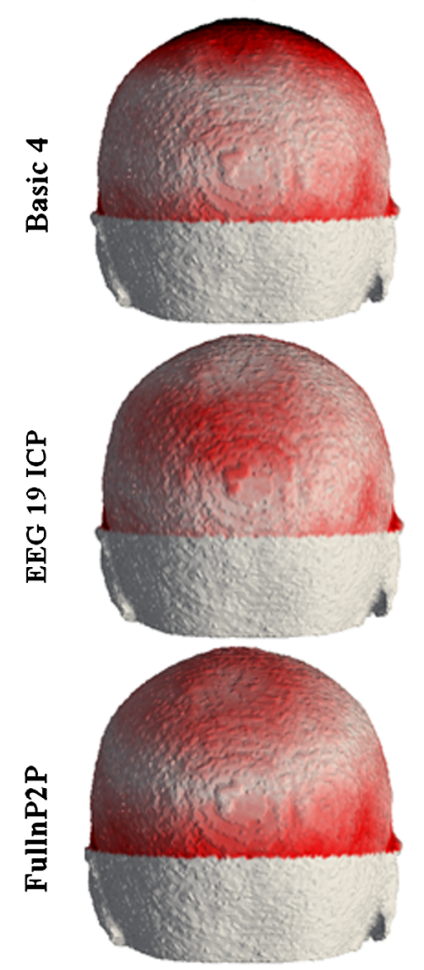

$0 \mathrm{~mm}$ (b)
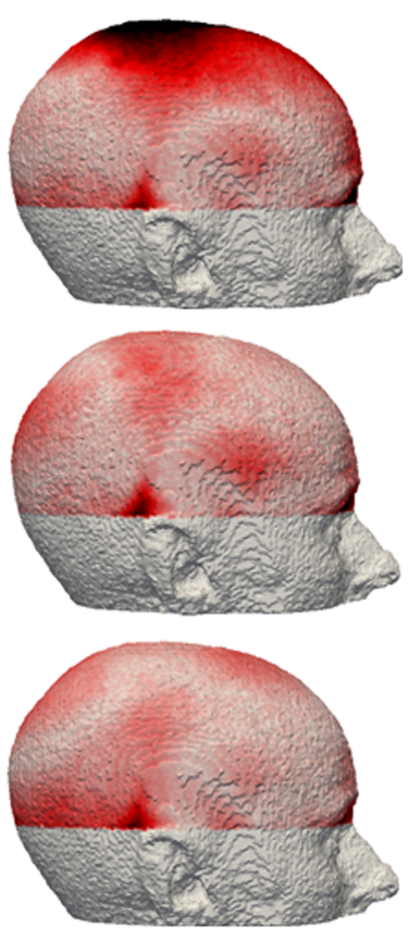

(c)
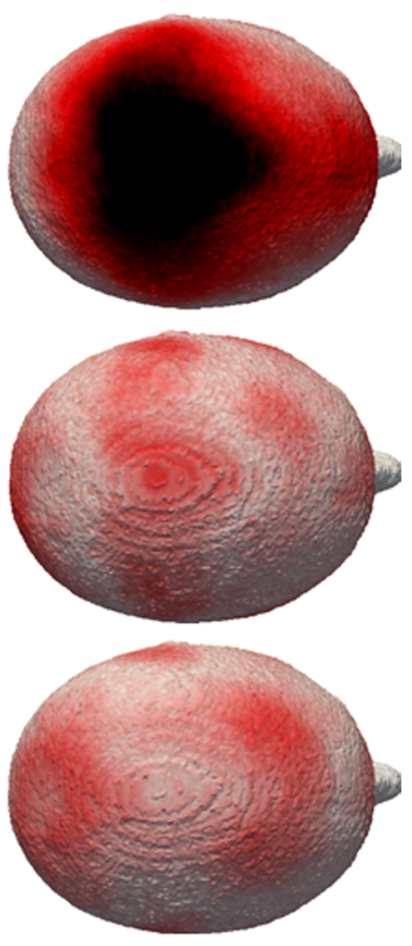

Fig. 4 An example of geometry error based on three registration methods for an example subject: (a) posterior view, (b) right view, and (c) top view. 


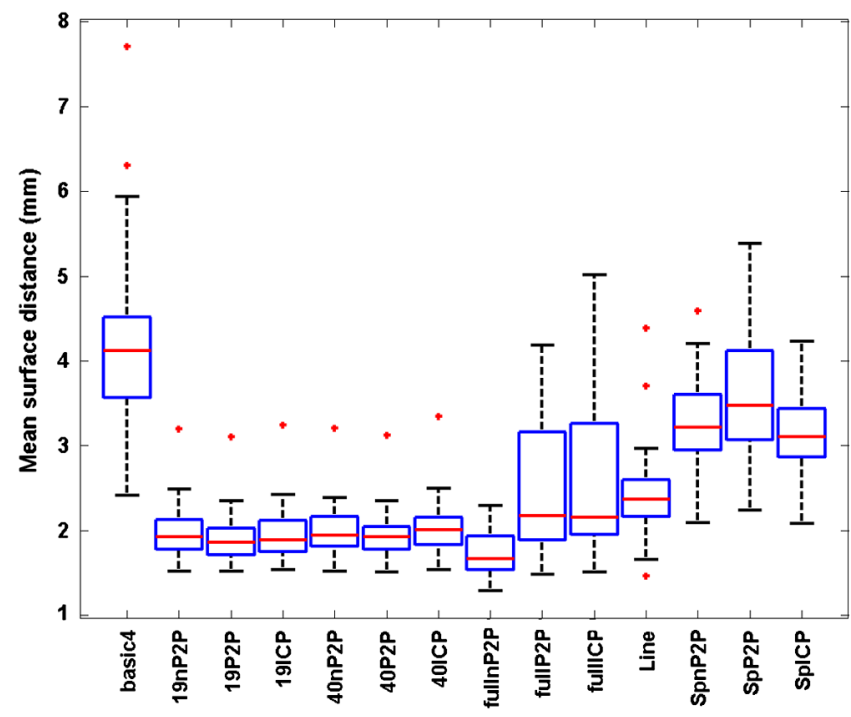

Fig. 5 Evaluation of geometrical errors based on 24 subjects. The central (red) lines represent the median, the box plots represent the 25th and 75th percentiles, whereas the whiskers present \pm 2.7 standard deviations. Outliers are presented as red crosses.

mesh onto the subject-specific mesh. The geometrical accuracy is defined as the external surface distances between the registered atlas and the subject-specific mesh on every surface node within the ROI. The geometrical error of three registration methods (basic-4-landmark registration, EEG 19 ICP registration, and full-head-landmark registration) for the same subject is shown in Fig. 4 as an example. As is evident, qualitatively, the basic-4-landmark registration method has the highest geometrical error $(\sim 10 \mathrm{~mm})$ among the three shown registration methods. For all shown registration methods, the error varies spatially: using the basic-4-landmark registration method, the upper middle surface area has a relatively high-geometrical error while the back and temple surface areas have a relatively low error. For the EEG 19 ICP registration method, the upper middle and the back surface area have a relatively high-geometrical error, whereas the front surface area has a relatively lowgeometrical error. For full-head-landmark system nP2P registration method, the upper front and upper back surface areas have a relatively high-geometrical error, whereas the lower side surface area has a relatively low-geometrical error.

The complete evaluation of the registration accuracy is based on the average surface distance of the registered atlas mesh for all 24 subjects as compared to the subject-specific mesh (Fig. 5). Of the utilized 14 registration methods, the full-head-landmark $\mathrm{nP} 2 \mathrm{P}$ registration method with $1.5( \pm 0.5) \mathrm{mm}$ average surface distance has the best average geometrical accuracy while the basic-4-landmark registration with $4( \pm 1) \mathrm{mm}$ average surface distance has the worst accuracy. The three 19 spherical coordinate landmarks-based registration with a $3.2( \pm 0.5) \mathrm{mm}$ average surface distance are the second least accurate registration methods. The line-fitting registration has a $2.3( \pm 0.5) \mathrm{mm}$ average surface distance. The full-head-landmark P2P and ICP registration methods have a $2.2 \mathrm{~mm}$ average surface distance, but they show a variation of $1.5 \mathrm{~mm}$, which is the largest difference among all subjects. The other six registration methods (EEG 19 and 40 landmark system with nP2P, P2P, and ICP registration methods) are less accurate with $2( \pm 0.5) \mathrm{mm}$ average surface distances.

\subsection{Geometry Accuracy of the Gray Matter}

Because of the error from the registration methods and the underlying differences between the internal structures of the atlas and subject-specific model, the final registration of the internal structure of the registered atlas mesh can also be inaccurate. This inaccuracy of the internal structure can be evaluated based on the geometrical analysis of gray matter registration itself. The geometrical accuracy of the gray matter is defined as the geometric distance between gray matter surfaces of the registered atlas mesh and the subject-specific mesh on each surface (gray matter) node. The surface nodes of the gray matter are selected for both the registered atlas mesh and the subject-specific mesh and the Euclidean distance is then calculated by the distance from each gray matter surface node of the subject-specific mesh and its closest gray matter surface node on the registered atlas mesh. As the geometrical accuracy varies in different areas of the gray matter, a structural regional map based on the previous studies and landmark structure such as the central sulcus and the lateral fissure is used on the cortex to aid spatial discrimination of the error seen in different areas (Fig. 6) ${ }^{49,50}$ This brain regional map contains four different lobes: the occipital, temporal, parietal, and frontal lobes, and it is used for a better analysis of the gray matter geometrical accuracy for different brain areas.

The geometrical accuracy of the gray matter registration for an example subject, based on three registration methods (basic4-landmark, EEG 19 ICP, and full-head-landmark), is shown in Fig. 7. For the entire gray matter surface within the ROI, the basic-4-landmark registration method has the lowest accuracy among the three registration methods with a $5 \mathrm{~mm}$ maximum surface distance. For all three registration methods shown, the geometrical accuracy of gray matter varies for different functional areas of the brain. For the basic-4-landmark registration method, the brain areas near the temporal, prefrontal, and occipital cortex regions have better accuracy as compared to others,

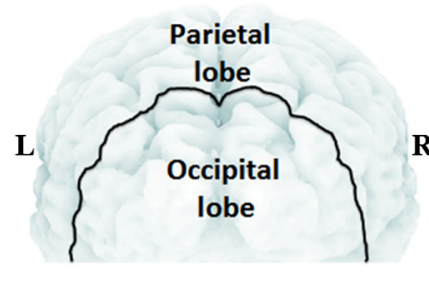

(a)

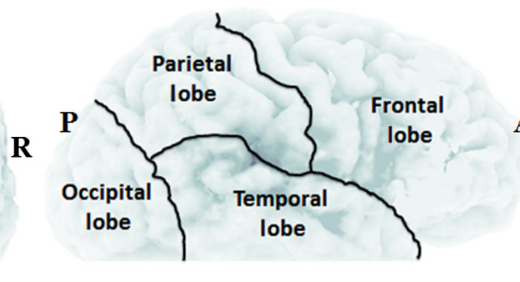

(b)

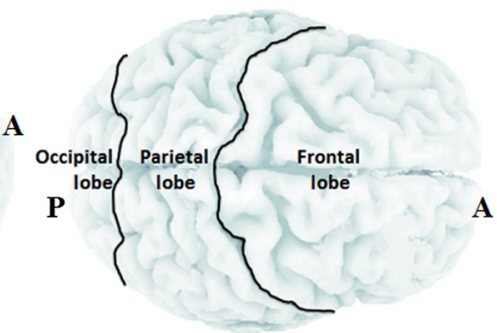

(c)

Fig. 6 Brain functional regions used for geometrical representation. (a) posterior view, (b) right view, and (c) top view. 
(a)
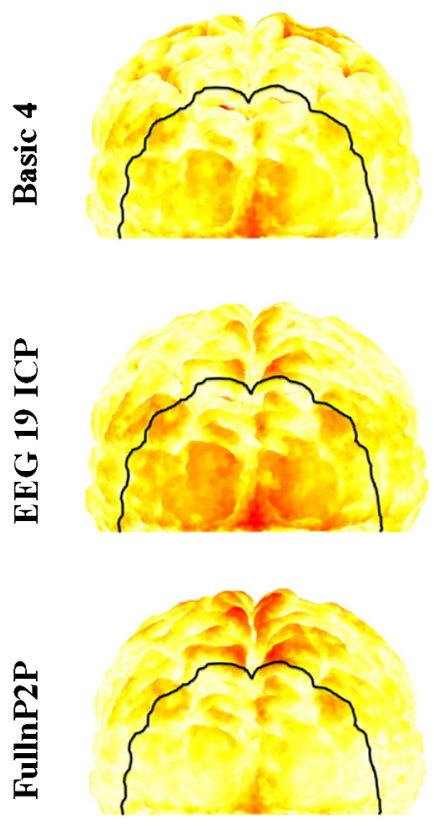

(b)
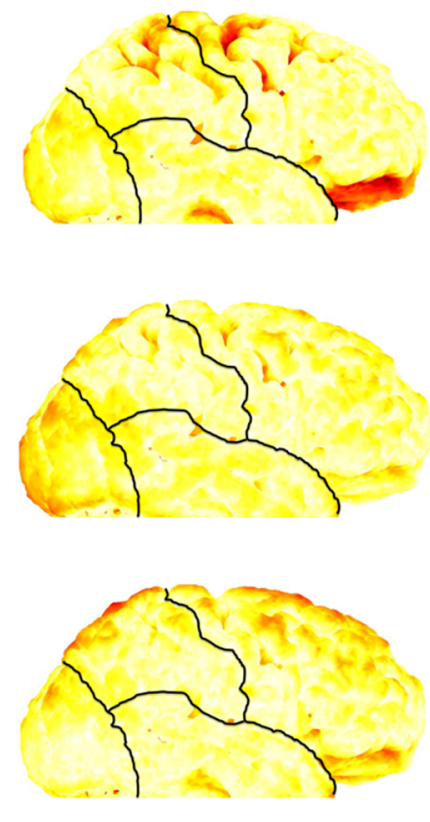

(c)
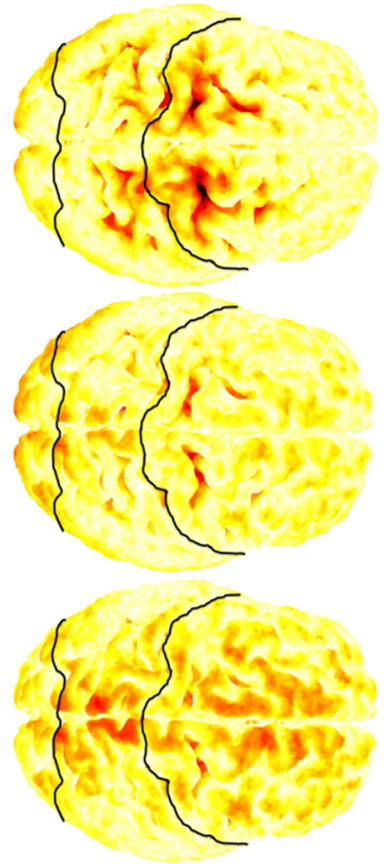

$0 \mathbf{~ m m}$

$5 \mathbf{~ m m}$

Fig. 7 An example of gray matter geometry errors based on three registration methods for an example subject: (a) posterior view, (b) right view, and (c) top view.

whereas the areas near the central cortex region (area adjoining frontal and parietal cortex regions) have the lowest accuracy. For the EEG 19 landmark system with ICP registration method, the areas near the temporal and prefrontal cortex regions have the best accuracy as compared to the other parts of the cortex, whereas the areas near the occipital cortex region have the lowest. For the full-head-landmark system with nP2P registration method, the areas near the occipital and temporal cortex regions have the best accuracy, whereas the areas near the superior frontal and superior parietal cortex regions have the lowest. However, due to the complex structure of the gray matter (such as the gyri), the gray matter surface accuracy may not fully represent the geometrical accuracy of the cortex registration itself.

\subsection{Evaluation of the Sensitivity Accuracy}

The accuracy of light propagation of the registered atlas mesh is evaluated based on the comparison between the sensitivity matrices from the subject-specific and the registered atlas mesh. The ROI for this evaluation is selected as the region within the gray matter with a sensitivity value higher than $1 \%$ of the maximum. Since the geometry of the subject-specific gray matter and the registered atlas gray matter will differ, some areas are excluded in the registered atlas mesh since there will be no common overlap in these areas. For the comparison, therefore, the sensitivity values of the registered atlas mesh, which have been excluded, are set as 0 .

As shown above, since the accuracy of geometrical registration varies for different brain regions, the sensitivity accuracy could also vary for different regions. The sensitivity errors of the cortex for one example subject, based on the three different registration methods are shown in Fig. 8. For all brain regions, the basic-4-landmark registration has the overall lowest sensitivity accuracy: the occipital cortex region has better accuracy as compared to other regions and the areas near the central cortex region have the lowest accuracy. For the EEG 19 landmark system with ICP registration method, the areas near the temporal and prefrontal cortex regions show better accuracy as compared to other regions, whereas the areas near the occipital and superior parietal cortex regions have a lower accuracy. For the full-head-landmark system with nP2P registration method, the areas near the temporal cortex region have a better accuracy as compared to other regions, whereas the areas near the superior frontal and superior parietal cortex regions have a lower accuracy. It is worth noting that the sensitivity accuracy distribution for different brain regions is similar to the geometrical accuracy distribution.

To fully quantify the evaluation of the sensitivity error for all 14 registration methods based on all 24 subjects, the sensitivity error across all brain regions is shown in Fig. 9. All registration methods have, on average, a sensitivity error of no more than $50 \%$. The full-head-landmark $\mathrm{nP} 2 \mathrm{P}$ registration method has a $32( \pm 8) \%$ average sensitivity error, which is the most accurate registration method based on the sensitivity accuracy. The line fitting registration and basic-4-landmark registration have $50( \pm 10) \%$ average sensitivity error, which are the least accurate methods. The three 19 spherical coordinates landmarks-based registrations have a $50( \pm 15) \%$ average sensitivity error and have lower accuracy as compared to the other registration methods. The full-head-landmark P2P and ICP registration methods have a $40( \pm 20) \%$ average sensitivity error, which shows the largest difference among all subjects. The other six registration methods (EEG 19 and 40 landmark system with nP2P, P2P, ICP registration methods) have similar accuracies with $35( \pm 5) \%$ average sensitivity error, which are more accurate than the full-head-landmark P2P and ICP registration methods.

Compared to other registration methods, the basic-4landmark registration and the three 19 spherical coordinates 
(a)
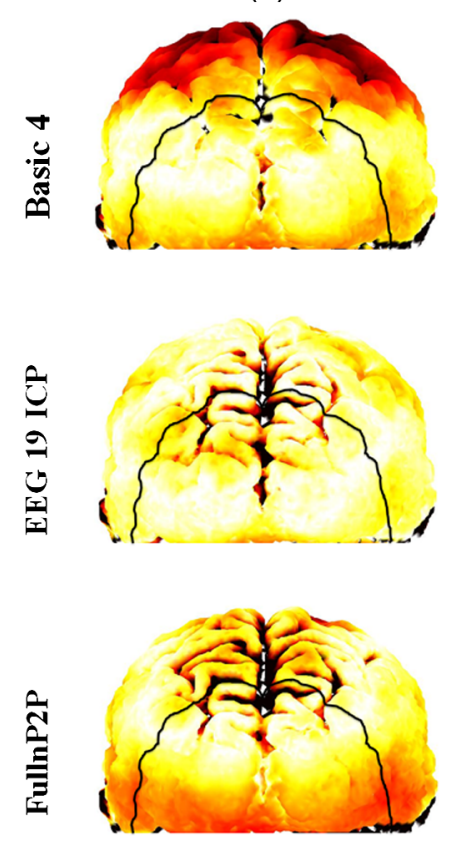

(b)

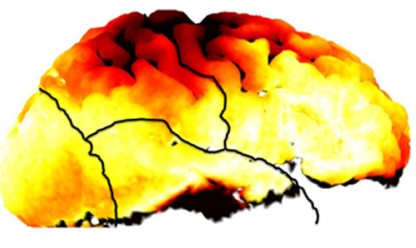

(c)

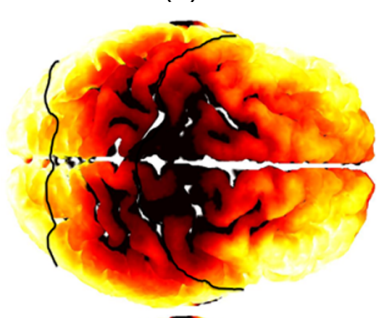

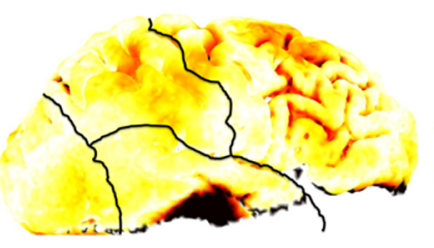
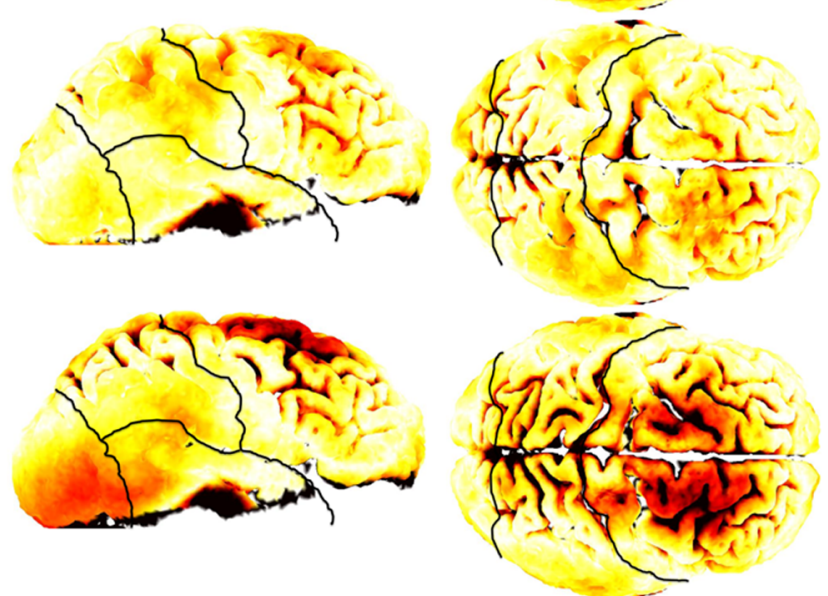

$100 \%$

Fig. 8 An example of sensitivity percentage error of the cortex based on three registration methods for an example subject: (a) posterior view, (b) right view, and (c) top view.

landmarks registration methods have a clear disadvantage for both geometrical and sensitivity accuracies. Therefore, for the remainder of the analysis, the three 19 spherical coordinatesbased landmarks registration methods are not considered, but since the basic-4-landmark registration relies on a minimum number of required landmarks, it will be included for analysis.

\subsection{Correlation Between Geometry and Sensitivity Accuracies}

Based on the analyses of the geometrical and sensitivity accuracies on the 24 subjects, there may exist some correlation

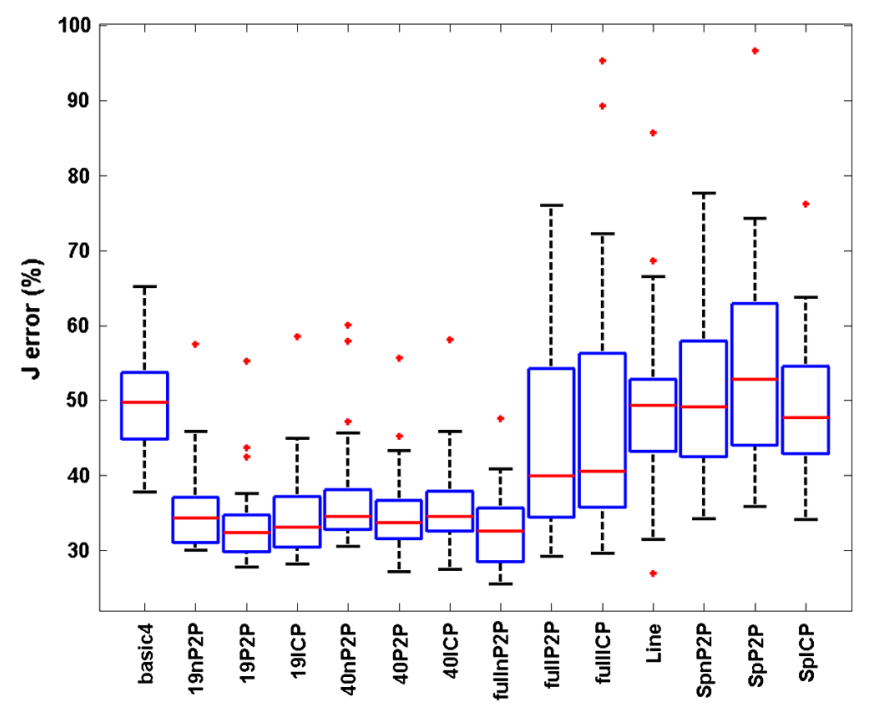

Fig. 9 Evaluation of sensitivity errors of the cortex based on 24 subjects. between these measures. A correlation analyses is performed for the registration methods between the geometrical and sensitivity accuracies on the whole head using the average surface distance error and the average sensitivity error (Fig. 10). As is evident, there is no strict linear relationship between the geometrical and sensitivity accuracies; however, the accuracy of the registration methods can be further classified. The full-headlandmark nP2P registration is considered as the most accurate method for both the geometrical and the sensitivity accuracies, and the basic-4-landmark registration is considered as the least accurate method. The full-head-landmark P2P and ICP

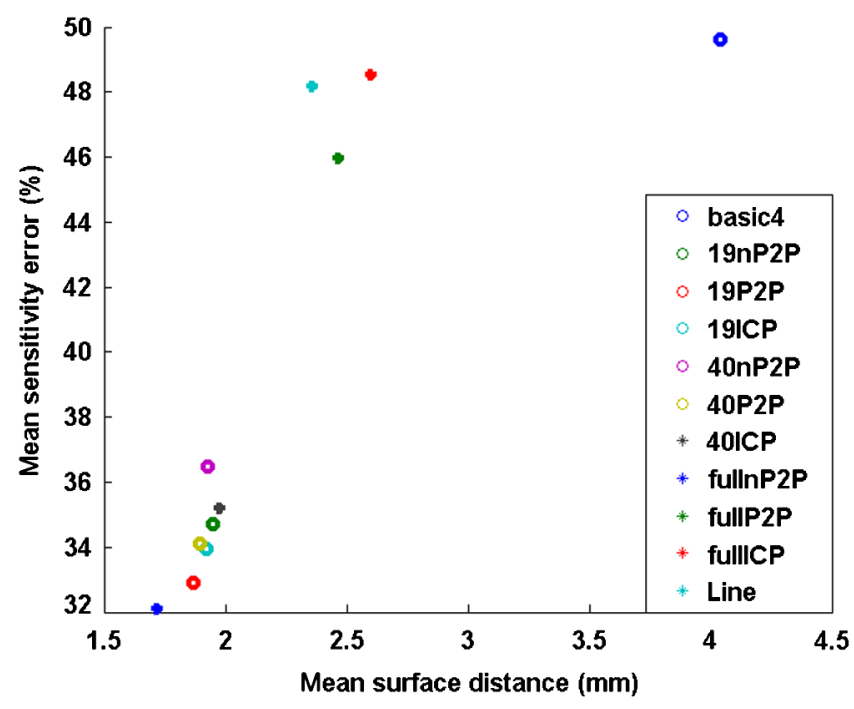

Fig. 10 Correlation between geometry error and sensitivity errors based on 24 subjects and registration methods. 

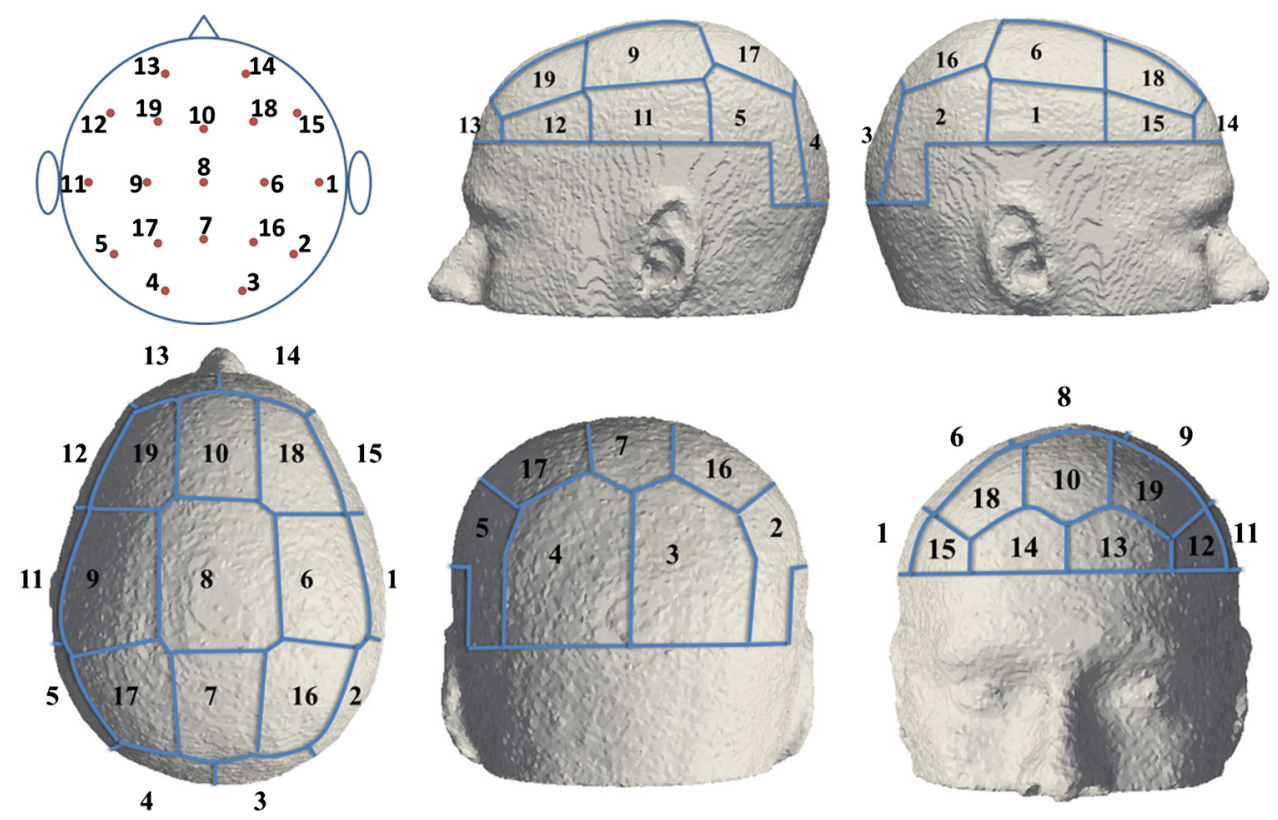

Fig. 11 Outline of the EEG 10/20-based head regions within the ROI for geometrical and sensitivity analysis.

registration and line-fitting registration methods have lower accuracy as compared to the other registration methods.

The analysis based on one example subject has shown that the geometrical and sensitivity accuracies can vary for different brain regions of the human brain. Therefore, there may be some classification for the correlation between these parameters on different brain regions. The 19 head regions within the ROI, based on the EEG 10/20 system, are used for the classification of the correlation of all 24 subjects (Fig. 11). The average geometrical and sensitivity accuracies for each subject are used for each region, separately, for all registration methods for all subjects and the correlation and strength (strength meaning magnitude, i.e., a higher strength would mean that a small change in one parameter will result in a large change in the other) between the geometrical and sensitivity accuracies are generated for each region. An example of these errors in relatively highly correlated and low correlated regions is shown in Figs. 12 and 13. For the high correlation region (region 2) with $R_{2}=0.95$, the basic-4landmark registration and full-head-landmark nP2P registration have the lowest geometrical error $(\sim 2 \pm 0.5 \mathrm{~mm})$. They also have the lowest sensitivity error $(\sim 35 \pm 7 \%)$. Line fitting registration has the highest geometrical error $(\sim 3 \mathrm{~mm})$ and sensitivity error $(\sim 60 \%)$. For this region, there is a clear linear relationship between the geometrical and the sensitivity accuracies for each of the registration methods. For the low correlation region (region 6) with $R_{2}=0.78$, full-head-landmark $\mathrm{nP} 2 \mathrm{P}$ registration has the lowest geometrical error $(\sim 1.5 \mathrm{~mm})$ and the lowest sensitivity error ( 30\%). But the EEG 19 and 40 landmark-based registration methods also have a relatively low-geometrical error $(\sim 1.7 \mathrm{~mm})$ and sensitivity error $(\sim 30 \%)$. In this region, there are no significant advantages in the accuracies of the geometrical and sensitivity among the registration methods. The full-head-landmark P2P and ICP registration and the linefitting registration have a $\sim 1.7( \pm 1) \mathrm{mm}$ geometry error and $\sim 40( \pm 20) \%$ sensitivity error, showing the largest accuracy difference across all of the 24 subjects. The basic-4-landmark registration with the highest geometrical error $(\sim 6.2 \mathrm{~mm})$ and the highest sensitivity error $(\sim 68 \%)$ has a clear disadvantage among all of the registration methods. However, there is no clear linear relationship between the geometrical and the sensitivity accuracies from the registration methods.

The correlation and strengths of all the 19 brain regions based on all of the 24 subjects with 11 of the registration methods are shown in Figs. 14 and 15. The correlation for the 19 regions varies from $R_{2}=0.7$ to $R_{2}=0.98$ and the strength for the 19 regions varies from 4 to 26 (the higher the strength, the higher the sensitivity error for a given geometrical error). Regions around the top of the head, which is near to the central cortex region, and the forehead, which is near the prefrontal cortex region, have a lower correlation and lower strength as compared to other head regions. Region 8 in the top middle part of the head has a correlation of $R_{2}=0.78$ and strength of 4 and it is one of the low correlation and low strength regions. Regions around the temples, which are near the temporal cortex region, have higher correlation and higher strengths as compared to other head regions. Region 2 near the right temple has a correlation of $R_{2}=0.98$ and strength of 26 and it is one of the highest correlation and high strength regions.

\section{Discussion}

Atlas-based DOT requires a subject-specific model based on the registration of the atlas model. The accuracy of the registration can directly affect the accuracy of the atlas-based model, and therefore, affect the accuracy of the simulated light propagation. Accuracy of the registration is evaluated using the geometrical accuracy of the registered atlas, and the accuracy of the light propagation is evaluated by the accuracy of the sensitivity matrix as generated from the registered atlas model.

Quantitative evaluation based on the whole head within the source-and-detector cap region using an HD cap is performed on 24 subjects and different rigid registration methods. Of these registration methods, 11 were based on either basic 4 or EEGbased landmarks and three were based on spherical coordinates as derived from three landmark systems. Of these, two different 

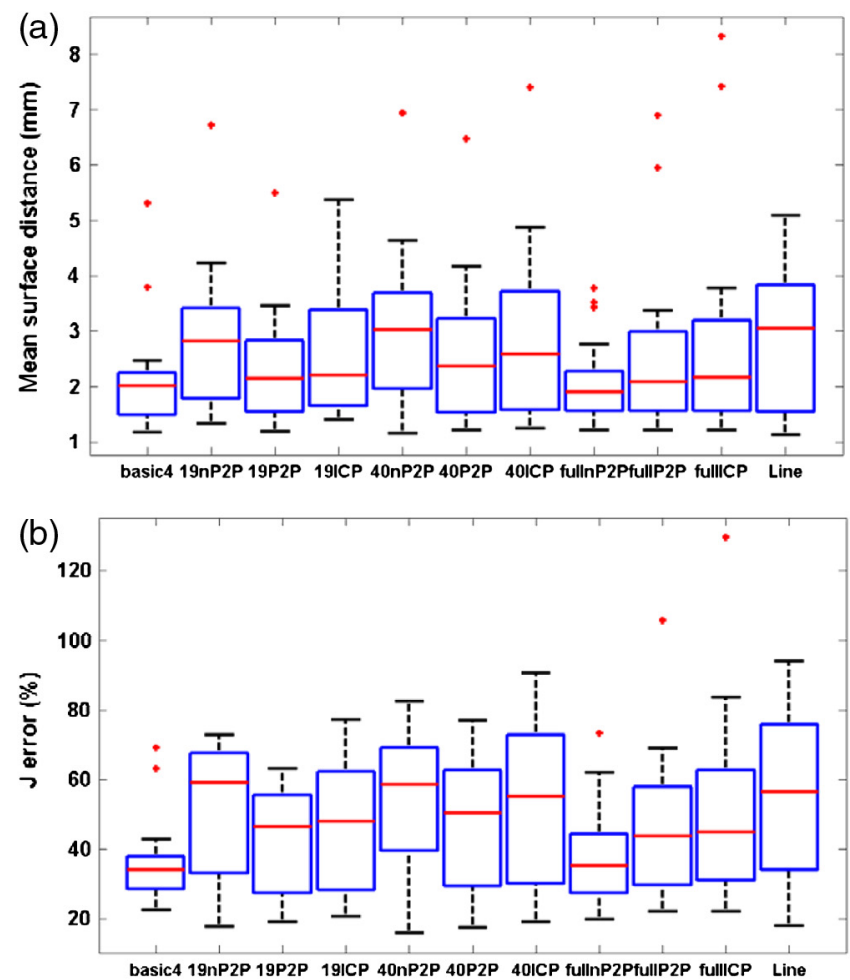

(c)

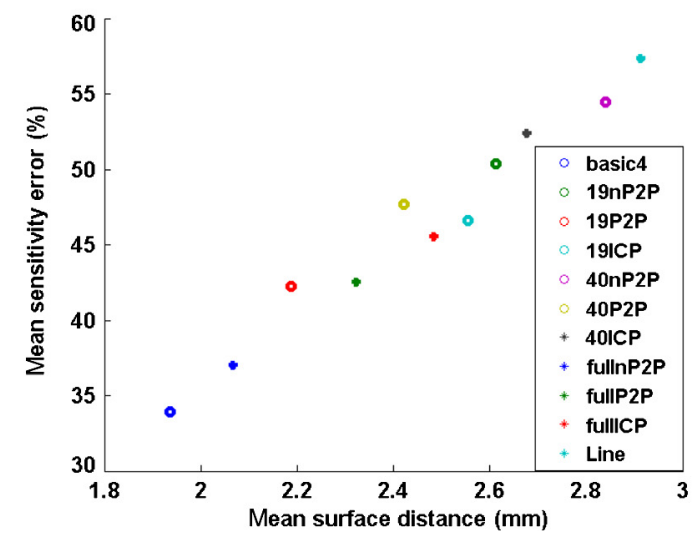

Fig. 12 Region 2 variation based on Fig. 11 showing a high correlation and medium strength (slope). (a) Evaluation of geometrical errors in region 2, (b) evaluation of sensitivity errors of the cortex in region 2, and (c) correlation between geometry error and sensitivity errors in region 2.

methods, generally the spherical coordinate landmark registration methods, even though in a practical setting they may be easier to define, did not perform as well as the EEG-based algorithms when considering the geometrical surface errors as well as the calculated sensitivity errors. This could be primarily due to the fact that using the spherical coordinates-based algorithms, landmarks are chosen arbitrarily and may not be best suited for registration as compared to well-defined EEG-based landmarks.

The full-head-landmark nP2P registration method has the most accurate method on both parameters (geometry and light propagation) among all registration methods. The line fitting registration and basic-4-landmark registration have the least accurate methods on the sensitivity with the line-fitting registration showing a slight advantage over the basic-4-landmark registration. The full-head-landmark P2P and ICP registration methods show the largest difference among different subjects
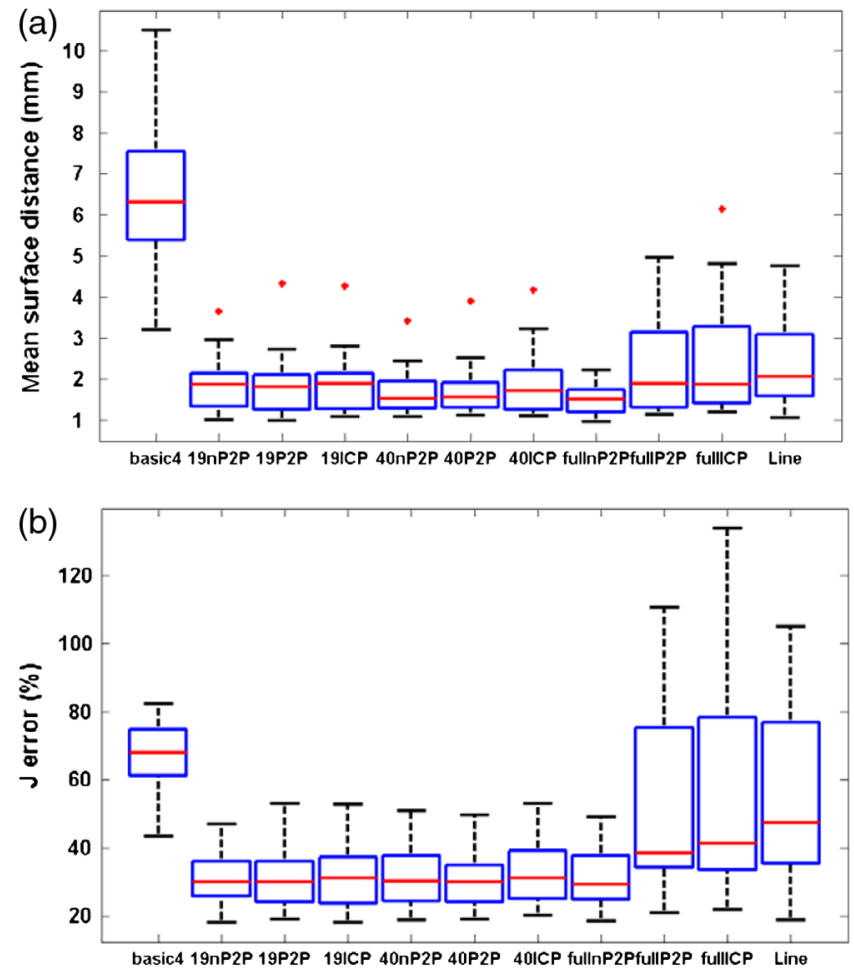

(c)

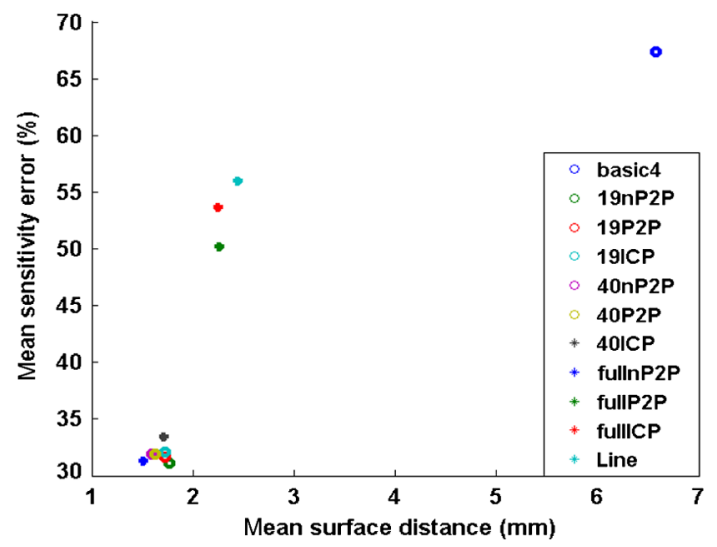

Fig. 13 Region 6 variation based on Fig. 11 showing a low correlation and high strength (slope). (a) Evaluation of geometrical errors in region 6 , (b) evaluation of sensitivity errors of the cortex in region 6 , and (c) correlation between geometry error and sensitivity errors in region 6 .

for both of the parameters evaluated. All other registration methods show similar accuracies and they are more accurate than either the full-head-landmark P2P or ICP registration methods based on both evaluations.

The accuracy of the registration is not uniformly distributed through different brain regions. The difference of accuracies between the regions can be caused by the distribution of the landmarks, which is the only basis of the optimization in the registration process. For example, the occipital cortex region contains one of the four landmarks in the basic-4-landmark system (the inion), which holds $25 \%$ of all landmarks in the registration. Because of this clear advantage over other regions, the occipital cortex region is one of the most accurately registered regions based on the registration method with basic-4-landmark system. However, the occipital cortex region does not show such an advantage when using a uniformly distributed landmark 

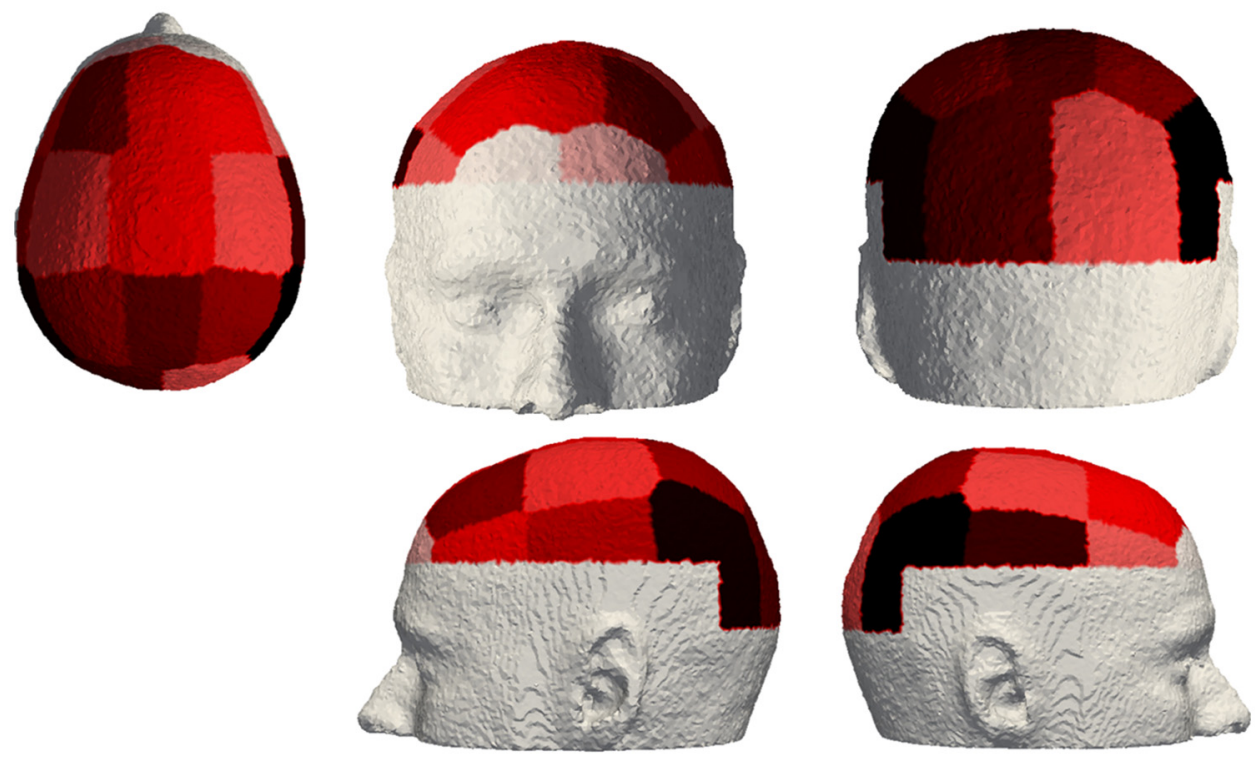

0.70

0.98

Fig. 14 Correlation between geometrical and sensitivity errors in all EEG 10/20-based head regions.

system such as the EEG 19 landmark system. In the EEG 19 landmark system, the occipital cortex region contains two of the 19 landmarks, which holds only $10.5 \%$ of all landmarks in the registration and it does not show a clear advantage over other regions. Furthermore, the location and extraction of the EEG 19 landmark can introduce additional spatial estimation errors ${ }^{51,52}$ which can also decrease the registration accuracy.

Although there is no clear linear relationship between the accuracies of geometry and light propagation, there are some similarities between the registration methods on both evaluation criteria. The results from the region-based correlation analyses of the two measures of accuracies shows that the correlation value $R_{2}$ varies from 0.7 to 0.98 through all of the defined
19 brain regions with most regions having a relatively high correlation. The two regions on the forehead show the lowest correlation value as these two regions contain some features which are hard to register. This can increase the geometrical inaccuracy in this region without large effects on the sensitivity accuracy.

Based on the analysis above, the most appropriate registration method varies for activities located in different functional brain regions. For example, the full-head-landmark nP2P registration method is the most accurate method for the central cortex region, whereas the basic-4-landmark registration method is the most accurate method for the temporal cortex region. Therefore, the registration method should be selected based on the location
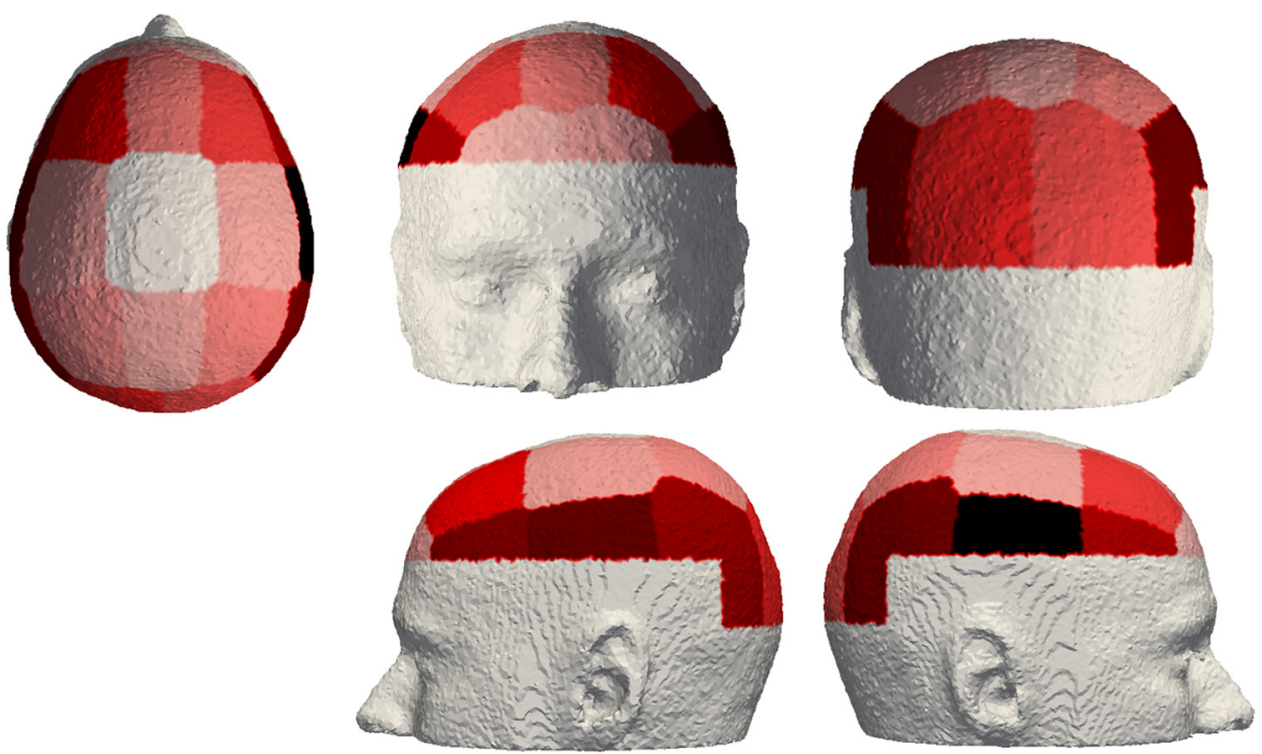

26

Fig. 15 Strength of geometrical and sensitivity errors in all EEG 10/20-based head regions. 
of the brain activities. For the whole cortex recovery, the fullhead-landmark nP2P registration method is the most accurate method. However, the extraction of the full-head-landmark and the registration process are more time consuming than the other registration methods. Since the EEG 19 registration-based methods with a small disadvantage in registration accuracy are more efficient as compared to the full-head-landmark nP2P registration method, it is the most appropriate registration method for the whole cortex recovery. Although there is little difference in the accuracy between EEG 19 ICP, EEG 19 P2P, and EEG 19 $\mathrm{nP} 2 \mathrm{P}$ registration methods, the iterative algorithms are more computationally demanding than the noniterative algorithms. Therefore, the EEG $19 \mathrm{nP} 2 \mathrm{P}$ registration method is a more efficient method as compared to EEG 19 P2P and EEG 19 ICP registration methods. We have previously shown that an error of $\sim 30 \%$ within the sensitivity matrix was acceptable for the recovery of focal activations from the visual cortex with less than a $4.50 \mathrm{~mm}$ accuracy in localization. ${ }^{34}$ It is, therefore, expected that similar results can be achieved for the whole cortex imaging using the EEG $19 \mathrm{nP} 2 \mathrm{P}$ registration method.

\section{Conclusions}

Atlas-based DOT in brain activation recovery, which is not constrained by the information of internal structure of the subject and relies only on the NIR data, is an emerging functional neuroimaging technology. The registration accuracy and its effect on the recovery result have been investigated in the past few years with studies which have been focused on the registration accuracy and recovery accuracy in localized areas. ${ }^{28-30}$ There are also studies of the comparison between nonrigid registration and rigid registration methods for the human head. ${ }^{40,53}$ In this paper, 19 rigid registration methods are evaluated and compared with the registration and the sensitivity accuracies are analyzed based on the whole head. It is shown that DOT recovery based on the atlas model and surface landmarks can provide a recovery result with an acceptable accuracy for the whole human cortex. It also demonstrates that a typical landmark-based registration method, such as EEG $19 \mathrm{nP} 2 \mathrm{P}$ registration, has an acceptable accuracy over the whole cortex region, but appropriate registration methods with a higher accuracy for the recovery of certain brain activation under investigation should be selected based on the functional brain regions involved.

\section{Acknowledgments}

This work has been funded by the National Institutes of Health Grant Nos. R01EB009233-2 and RO1-CA132750 and Autism Speaks Meixner Translational Postdoctoral Fellowship 7962.

\section{References}

1. P. T. Fox et al., "Mapping human visual cortex with positron emission tomography," Nature 323, 806-809 (1986).

2. K. K. Kwong et al., "Dynamic magnetic resonance imaging of human brain activity during primary sensory stimulation," Proc. Natl. Acad. Sci. U. S. A. 89, 5675-5679 (1992).

3. J. W. Belliveau et al., "Functional mapping of the human visual cortex by magnetic resonance imaging," Science 254, 716-719 (1991).

4. M. E. Raichle, "Behind the scenes of functional brain imaging: a historical and physiological perspective," Proc. Natl. Acad. Sci. U. S. A. 95, 765-772 (1998).

5. S. Arridge and M. Schweiger, "Gradient-based optimisation scheme for optical tomography," IEEE Trans. Med. Imaging 18, 262-271 (1999).
6. A. H. Hielscher, A. D. Klose, and K. M. Hanson, "Gradient-based iterative image reconstruction scheme for time-resolved optical tomography," IEEE Trans. Med. Imaging 18, 262-271 (1999).

7. B. Biswal et al., "Functional connectivity in the motor cortex of resting human brain using echo-planar MRI," Magn. Reson. Med. 34, 537-541 (1995).

8. A. C. Nobre et al., "Functional localization of the system for visuospatial attention using positron emission tomography," Brain 120, 515-533 (1997).

9. T. Matsuda et al., "Functional MRI mapping of brain activation during visually guided saccades and antisaccades: cortical and subcortical networks," Psychiatry Res. 131, 147-155 (2004).

10. A. T. Eggebrecht et al., "Mapping distributed brain function and networks with diffuse optical tomography," Nat. Photonics 8, 448-454 (2014).

11. E. Courchesne et al., "Mapping early brain development in autism," Neuron 56, 399-413 (2007).

12. M. Mesulam, "Defining neurocognitive networks in the BOLD new world of computed connectivity," Neuron 62, 1-3 (2009).

13. V. Menon, "Large-scale brain networks and psychopathology: a unifying triple network model," Trends Cognit. Sci. 15, 483-506 (2011).

14. K. A. Pelphrey et al., "Research review: constraining heterogeneity: the social brain and its development in autism spectrum disorder," J. Child Psychol. Psychiatry Allied Discip. 52, 631-644 (2011).

15. B. R. White et al., "Resting-state functional connectivity in the human brain revealed with diffuse optical tomography," Neuroimage 47, 148156 (2009).

16. M. E. Phelps and J. C. Mazziotta, "Positron emission tomography: human brain function and biochemistry," Science 228, 799-809 (1985).

17. J. Sergent, S. Ohta, and B. MacDonald, "Functional neuroanatomy of face and object processing. A positron emission tomography study," Brain 115(Pt 1), 15-36 (1992).

18. S. A. Engel, G. H. Glover, and B. A. Wandell, "Retinotopic organization in human visual cortex and the spatial precision of functional MRI," Cereb. Cortex 7, 181-192 (1997).

19. M. Wolf, M. Ferrari, and V. Quaresima, "Progress of near-infrared spectroscopy and topography for brain and muscle clinical applications," J. Biomed. Opt. 12, 062104 (2007).

20. F. Irani et al., "Functional near infrared spectroscopy (fNIRS): an emerging neuroimaging technology with important applications for the study of brain disorders," Clin. Neuropsychol. 21, 9-37 (2007).

21. F. Scholkmann et al., "A review on continuous wave functional nearinfrared spectroscopy and imaging instrumentation and methodology," Neuroimage 85, 6-27 (2014).

22. A. Torricelli et al., "Time domain functional NIRS imaging for human brain mapping," Neuroimage 85(Pt 1), 28-50 (2014).

23. D. Tsuzuki and I. Dan, "Spatial registration for functional near-infrared spectroscopy: from channel position on the scalp to cortical location in individual and group analyses," Neuroimage 85(Pt 1), 92-103 (2014).

24. H. Dehghani et al., "Depth sensitivity and image reconstruction analysis of dense imaging arrays for mapping brain function with diffuse optical tomography," Appl. Opt. 48, D137-D143 (2009).

25. A. P. Gibson, J. C. Hebden, and S. R. Arridge, "Recent advances in diffuse optical imaging," Phys. Med. Biol. 50, R1-R43 (2005).

26. Y. Zhan et al., "Image quality analysis of high-density diffuse optical tomography incorporating a subject-specific head model," Front. Neuroenerg. 4, 6 (2012).

27. C. Habermehl et al., "Somatosensory activation of two fingers can be discriminated with ultrahigh-density diffuse optical tomography," Neuroimage 59, 3201-3211 (2012).

28. A. Custo et al., "Anatomical atlas-guided diffuse optical tomography of brain activation," Neuroimage 49, 561-567 (2010).

29. R. J. Cooper et al., "Validating atlas-guided DOT: a comparison of diffuse optical tomography informed by atlas and subject-specific anatomies," Neuroimage 62, 1999-2006 (2012).

30. S. L. Ferradal et al., "Atlas-based head modeling and spatial normalization for high-density diffuse optical tomography: in vivo validation against fMRI," Neuroimage 85(Pt 1), 117-126 (2014).

31. P. Giacometti, K. L. Perdue, and S. G. Diamond, "Algorithm to find high density EEG scalp coordinates and analysis of their correspondence to structural and functional regions of the brain," J. Neurosci. Methods 229, 84-96 (2014). 
32. H. Dehghani et al., "Near infrared optical tomography using NIRFAST: algorithm for numerical model and image reconstruction," Coтmun. Numer. Methods Eng. 25, 711-732 (2009).

33. K. J. Friston, "Introduction: experimental design and statistical parametric mapping," in Human Brain Function, 2nd ed., R. S. J. Frackowiak et al., Eds., Academic Press (2003).

34. J. Ashburner and K. J. Friston, "Image segmentation," in Human Brain Function, 2nd ed., R. S. J. Frackowiak et al., Eds., Academic Press (2003).

35. M. Jermyn et al., "Fast segmentation and high-quality three-dimensional volume mesh creation from medical images for diffuse optical tomography," J. Biomed. Opt. 18, 086007 (2013).

36. A. T. Eggebrecht et al., "A quantitative spatial comparison of highdensity diffuse optical tomography and fMRI cortical mapping," Neuroimage 61, 1120-1128 (2012).

37. G. Strangman, M. A. Franceschini, and D. A. Boas, "Factors affecting the accuracy of near-infrared spectroscopy concentration calculations for focal changes in oxygenation parameters," Neuroimage 18, 865-879 (2003).

38. A. Custo et al., "Effective scattering coefficient of the cerebral spinal fluid in adult head models for diffuse optical imaging," Appl. Opt. 45, 4747-4755 (2006).

39. F. Bevilacqua et al., "In vivo local determination of tissue optical properties: applications to human brain," Appl. Opt. 38, 4939-4950 (1999).

40. A. Klein et al., "Evaluation of 14 nonlinear deformation algorithms applied to human brain MRI registration," Neuroimage 46, 786-802 (2009).

41. X. Wu et al., "Quantitative evaluation of atlas-based high-density diffuse optical tomography for imaging of the human visual cortex," Biomed. Opt. Express 5, 3882-3900 (2014).

42. V. L. Towle et al., "The spatial location of EEG electrodes: locating the best-fitting sphere relative to cortical anatomy," Electroencephalogr. Clin. Neurophysiol. 86, 1-6 (1993).
43. T. D. Lagerlund et al., "Determination of 10-20 system electrode locations using magnetic-resonance image scanning with markers," Electroencephalogr. Clin. Neurophysiol. 86, 7-14 (1993).

44. D. Tsuzuki et al., "Stable and convenient spatial registration of standalone NIRS data through anchor-based probabilistic registration," Neurosci. Res. 72, 163-171 (2012).

45. J. Mazziotta et al., "A four-dimensional probabilistic atlas of the human brain," J. Am. Med. Inf. Assoc. 8, 401-430 (2001).

46. J. Mazziotta et al., "A probabilistic atlas and reference system for the human brain: International Consortium for Brain Mapping (ICBM)," Philos. Trans. R. Soc. B 356, 1293-1322 (2001).

47. S. L. Ferradal et al., "Functional imaging of the developing brain at the bedside using diffuse optical tomography," Cereb. Cortex bhu320 (2015).

48. M. E. Eames et al., "An efficient Jacobian reduction method for diffuse optical image reconstruction," Opt. Express 15, 15908-15919 (2007).

49. N. Tzourio-Mazoyer et al., "Automated anatomical labeling of activations in SPM using a macroscopic anatomical parcellation of the MNI MRI single-subject brain," Neuroimage 15, 273-289 (2002).

50. D. Pantazis et al., "Comparison of landmark-based and automatic methods for cortical surface registration," Neuroimage 49, 2479-2493 (2010).

51. M. Okamoto et al., "Three-dimensional probabilistic anatomical craniocerebral correlation via the international 10-20 system oriented for transcranial functional brain mapping," Neuroimage 21, 99-111 (2004).

52. V. Jurcak, D. Tsuzuki, and I. Dan, "10/20, 10/10, and 10/5 systems revisited: their validity as relative head-surface-based positioning systems," Neuroimage 34, 1600-1611 (2007).

53. B. A. Ardekani et al., "Quantitative comparison of algorithms for intersubject registration of 3D volumetric brain MRI scans," J. Neurosci. Methods 142, 67-76 (2005).

Biographies for the authors are not available. 